Christian Larsen

Derivation of confidence intervals of service measures in a base-stock inventory control system with low-frequent demand

CORAL - Centre for Operations

Research Applications in Logistics 


\title{
Derivation of confidence intervals of service measures in a base-stock inventory control system with low-frequent demand
}

\author{
Christian Larsen \\ Centre for Operations Research Applications in Logistics, Department of Business Studies, Aarhus School of \\ Business, University of Aarhus, Fuglesangs Allé 4, DK-8210 Aarhus V, Denmark. \\ chl@asb.dk
}

\begin{abstract}
We explore a base-stock system with backlogging where the demand process is a compound renewal process and the compound element is a delayed geometric distribution. For this setting it is proven in [4] that the long-run average service measures order fill rate $(O F R)$ and volume fill rate $(V F R)$ are equal in values. In [4] it is also demonstrated that although equal ex ante one will ex post observe differences as actual sample paths are different. By including a low-frequency assumption in the model, we are able to derive mathematical expressions of the confidence intervals one will get if $O F R$ and $V F R$ are estimated in a simulation using the regenerative method. Through numerical examples we show that of the two service measures it is $O F R$ that can be estimated most accurately. However, simulation results show that the opposite conclusion holds if we instead consider finitehorizon service measures, namely per-cycle variants of $O F R$ and $V F R$.

Keywords: Inventory control, base-stock systems, confidence intervals of service measures.
\end{abstract}

\section{Introduction}

It is a well recognized approach in both academia and practice to apply service measures to evaluate the performance of an inventory system. Typically, these service measures are expressed as average values. Thus, they do not give any information about how much the deviation (from this predicted average value) actually will be. This means that when setting control parameters ex ante, based on expected service levels, and then estimating performance ex post, say after a year, one might very well observe some deviations between estimated service levels and predicted service levels. It would be of interest to classify various service measures according to how much actual realizations might differ from predicted values. This calls for not solely focusing on predicted values but also on deriving measures of variance of the service measures. This is the principal goal of the paper. We explore this issue in a basestock system with backlogging where the demand process is a compound renewal process and the compound element is a delayed geometric distribution. For this setting it is proven in [4] that the long-run average service measures order fill rate $(O F R)$ and volume fill rate $(V F R)$ are equal. But this result only holds when considering their mean values as actual sample paths are different. This is demonstrated in a simulation study in [4] (see Table 5 therein). By comparing the reported confidence intervals in that table, it can be seen that OFR has the narrowest interval. The table also indicates that it seems easier to achieve the pre-specified service level target by using the $O F R$ service measure. It is difficult mathematically to derive higher order moments of service measures. However, by including a low-frequency assumption in the model of [4], we are able to derive a measure of the variance of the two service measures. Specifically, we derive mathematically the confidence interval of $O F R$ and $V F R$ if these are estimated in a simulation by the regenerative method. In Section 2 we first give mathematical derivations of the service measures $O F R$ and $V F R$. These derivations explicitly exploit the low-frequency assumptions and therefore appear as alternative derivations compared to [4]. Then in Section 3 we mathematically derive the confidence intervals of our service measures. In Section 4 we present numerical examples from which it can be concluded that it seems to be $O F R$ that can be estimated most accurately. In Section 4 we also explore what happens if we instead of long-run average service measures look at percycle service measures (denoted respectively $O F R_{C y c l e}$ and $V F R_{C y c l e}$ ); these can be considered a sort of finite-horizon service measure. The study of finite-horizon service measures has not received much attention in the literature. There are some analyses of the finite-horizon 
volume fill rate service measure in [2] and [8]. Also the minimal service level constraints studied in [1] can be viewed as a finite-horizon service measure. By means of a simulation experiment, we show that for a given base-stock parameter, the per-cycle service levels are higher than their long-run counterparts. This result is in accordance with [2], which proved its result under more restrictive assumptions. Furthermore, of the two per-cycle service measures, it is $V F R_{\text {Cycle }}$ that can be estimated most accurately. Finally, Section 5 contains some concluding remarks.

\section{Derivation of the (long-run) average service measures}

We consider a base-stock inventory control system with parameter $S$ which is a positive integer. All replenishment orders are issued instantaneously at the time point of an order request and all replenishments have a constant lead time $L$. All unfilled demand is backlogged and serviced as soon as possible. Any customer order can be modelled as a positive integer valued random variable $X$. We assume no correlation between the sizes of successive customer orders. Let $X_{0}, X_{1}, \ldots$ be a sequence of independent random variables (denoting successive customer orders) having the same distribution as $X$. The inter-arrival times between order requests are also assumed to be independent. Thus, any inter-arrival time can be modelled as a positive continuous random variable $T$. We also assume that $T$ and $X$ are independent. Let $T_{1}, T_{2}, \ldots$ be a sequence of independent random variables (denoting successive inter-arrival times) having the same distribution as $T$. We will assume that the demand process is low-frequent. This characteristic, also denoted intermittent, is often encountered for inventories of spare parts; see for instance [3,6,9]. Specifically, we assume that

$$
P(T>L / 2)=1 \text {. }
$$

This means that at most one new customer order will appear during the replenishment leadtime of any order. As an example consider that $L=5$ and $T$ is uniformly distributed between 4 and 9 . Then (1) holds and $P(T<L)=0.2$. Thus, during any replenishment there is a probability of 0.2 that one (and only one) new customer order will appear. Assumption (1) also implies that upon the arrival of any customer order there is at most one replenishment order not yet received at the stocking point. Define a regeneration point to be the time point where the net inventory (the net inventory is the physical on-hand inventory minus the amount backlogged, see [7] p. 233) is $S$, but a new customer order has just appeared. We call this order the triggering order and represent its size by the random variable $X_{0}$. Denote by $N$ the number of orders (except the triggering order) that occurs between two successive regeneration points (which is called a cycle). The random variable $N$ has the following probability distribution

$$
P(N=i)=P(T \geq L)(P(T<L))^{i} \quad i=0,1,2 \ldots
$$

Thus, $N$ is geometrically distributed with mean $E[N]=P(T<L) / P(T \geq L)$. In Figure 1 we have illustrated a cycle in the case where $N=2$.

$<$ Figure 1 about here $>$

Define the random variables (the random variable $I_{\{A\}}$ is 1 if the event $A$ occurs and 0 otherwise): 


$$
\begin{aligned}
& O_{0}=I_{\left\{X_{0} \geq S+1\right\}}, \\
& O_{i}=I_{\left\{X_{i-1}+X_{i} \geq S+1\right\}} \quad i=1,2, \ldots, \\
& B_{0}=\max \left\{X_{0}-S, 0\right\}
\end{aligned}
$$

and

$$
B_{i}=\max \left\{\min \left\{X_{i-1}, S\right\}+X_{i}-S, 0\right\} \quad i=1,2 \ldots
$$

As stated previously, we will focus on the two service measures volume fill rate (VFR), and order fill rate $(O F R)$ defined as follows:

$1-O F R=\{$ The expected number of orders in a cycle where the whole order is not delivered immediately $) /\{$ The expected number of orders in a cycle $\}$

and

$1-V F R=\{$ The expected accumulated backlog in a cycle $\} /\{$ The expected demand in a cycle $\}$

The trigger order, order number 0 of the cycle, causes a backlog if $X_{0} \geq S+1$ and it causes an expected backlog $E\left[\max \left\{X_{0}-S, 0\right\}\right]$. Order number $i$ of the cycle $(i \geq 1)$ is not filled entirely if $X_{i-1}+X_{i} \geq S+1$ and it generates an expected backlog $E\left[\max \left\{\min \left\{X_{i-1}, S\right\}+X_{i}-S, 0\right\}\right]$. Therefore,

$$
1-O F R=\frac{E\left[O_{0}\right]+E\left[\sum_{i=1}^{N} O_{i}\right]}{1+E[N]}
$$

and

$$
1-V F R=\frac{E\left[B_{0}\right]+E\left[\sum_{i=1}^{N} B_{i}\right]}{E\left[X_{0}\right]+E\left[\sum_{i=1}^{N} X_{i}\right]} .
$$

For the general case, without (1), OFR and VFR are derived in a different manner in [3]. In Appendix A, we sketch how (7) and (8) could have been derived using the way of reasoning of [4]. In [4] it is shown that $V F R=O F R$ when the order sizes are independent and delayed geometric distributed. That is, $X$ has probability distribution

$$
P(X=j)=(1-\rho) \rho^{j-1} \quad j=1,2, \ldots
$$


where the probability parameter $\rho$ is between 0 and 1 . The term "delayed" is used because the distribution is specified for positive integers rather than for non-negative integers as for example in (2). For our specific case, assuming (1), we get

$$
1-O F R=1-V F R=\frac{\rho^{S}(1+E[N](1-S))+S E[N] \rho^{S-1}}{E[N]+1} .
$$

We have made a derivation of (10) in Appendix B.

\section{Deriving measures of variance of the service measures}

According to the theory about the regenerative method for estimating performance measures, see pp. 557-559 in [5], one should consider the random variables $D_{O F R}$ and $D_{V F R}$ defined as

$$
D_{\text {OFR }}=O_{0}+\sum_{i=1}^{N} O_{i}-(1-O F R)[1+N]
$$

and

$$
D_{V F R}=B_{0}+\sum_{i=1}^{N} B_{i}-(1-V F R)\left[X_{0}+\sum_{i=1}^{N} X_{i}\right] .
$$

According to [5] the half-width of the $100(1-\alpha) \%$ confidence interval of the estimated service measures is then given by

$$
z_{1-\alpha / 2} \frac{\sqrt{V\left[D_{O F R}\right]}}{(E[N]+1) \sqrt{n}}
$$

and

$$
z_{1-\alpha / 2} \frac{\sqrt{V\left[D_{V F R}\right]}}{(E[N]+1) E[X] \sqrt{n}}
$$

where $n$ is the number of cycles observed in the simulation, and $z_{1-\alpha / 2}$ is the upper $1-\alpha / 2$ critical point of the unit (mean 0 and standard deviation 1 ) normal distribution. Therefore, we define that service measure $O F R$ can be estimated most accurately if

$$
V\left[D_{O F R}\right]<\frac{V\left[D_{V F R}\right]}{(E[X])^{2}}
$$

Similarly, we define that $V F R$ can be estimated most accurately if (15) holds with the inequality sign reversed. From Appendix $\mathrm{C}$ and $\mathrm{D}$ and (10) it can be derived that $V\left[D_{\text {OFR }}\right]$ can be written in the following way:

$$
V\left[D_{\text {OFR }}\right]=\beta_{1} \rho^{S-1}+\beta_{2} \rho^{S}+\beta_{3} \rho^{2 S-2}+\beta_{4} \rho^{2 S-1}+\beta_{5} \rho^{2 S} .
$$


The coefficients $\beta_{1}, \ldots, \beta_{5}$ are given as follows:

$$
\begin{gathered}
\beta_{1}=(S+2) E[N]-2 P(N \geq 1), \\
\beta_{2}=1+(3-S) E[N] \\
\beta_{3}=S^{2}\left\{-3 E[N]+2 P(N \geq 1)+E\left[N^{2}\right]\right. \\
\left.+\left(\frac{E[N]}{E[N]+1}\right)^{2} E\left[(N+1)^{2}\right]-2 \frac{E[N]}{E[N]+1} E[N(N+1)]\right\} \\
\left.\beta_{4}=\left(6 S^{2}-4 S-2\right)\right) E[N]+2\left(-2 S^{2}+S+1\right) P(N \geq 1)+2 S(1-S) E\left[N^{2}\right] \\
+\frac{2 E[N] S(1+E[N](1-S))}{(E[N]+1)^{2}} E\left[(N+1)^{2}\right] \\
-2 \frac{1+E[N](1-S)}{1+E[N]} S E[N(N+1)] \\
-2 \frac{E[N]}{1+E[N]} S\left(1+(2-S) E[N]+(1-S) E\left[N^{2}\right]\right)
\end{gathered}
$$

and

$$
\begin{gathered}
\beta_{5}=\left(-3 S^{2}+4 S-1\right) E[N]+2 S(S-1) P(N \geq 1)+(1-S)^{2} E\left[N^{2}\right] \\
+\left(\frac{1+(1-S) E[N]}{1+E[N]}\right)^{2} E\left[(N+1)^{2}\right] \\
-2 \frac{1+(1-S) E[N]}{1+E[N]}\left(1+(2-S) E[N]+(1-S) E\left[N^{2}\right]\right) .
\end{gathered}
$$

Similarly, from Appendix $\mathrm{C}$ and $\mathrm{E}$ and (10) it can be derived that $V\left[D_{V F R}\right]$ can be written in the following way:

$$
V\left[D_{V F R}\right]=\frac{\gamma_{1} \rho^{S-1}+\gamma_{2} \rho^{S}+\gamma_{3} \rho^{S+1}+\gamma_{4} \rho^{2 S-2}+\gamma_{5} \rho^{2 S-1}+\gamma_{6} \rho^{2 S}+\gamma_{7} \rho^{2 S+1}}{(1-\rho)^{2}} .
$$

The coefficients $\gamma_{1}, \ldots, \gamma_{7}$ are given as follows:

$$
\begin{aligned}
& \gamma_{1}=(S+4) E[N]-4 P(N \geq 1), \\
& \gamma_{2}=1+3 E[N], \\
& \gamma_{3}=1+(1-S) E[N],
\end{aligned}
$$




$$
\begin{gathered}
\gamma_{4}=-S(2 S+3) E[N]+S(S+3) P(N \geq 1)+S^{2} E\left[N^{2}\right] \\
+\left(\frac{S E[N]}{1+E[N]}\right)^{2} E\left[(N+1)^{2}\right]-\frac{2 S^{2} E[N]\left(S E[N]+E\left[N^{2}\right]\right)}{1+E[N]} \\
\gamma_{5}=4\left(S^{2}-1\right) E[N]+2\left(-S^{2}-S+2\right) P(N \geq 1)+2 S(1-S) E\left[N^{2}\right] \\
+\frac{S E[N]\left(S E[N](1+E[N])+2(1+(1-S) E[N]) E\left[(N+1)^{2}\right]\right)}{(1+E[N])^{2}} \\
-\frac{2(1+(1-S) E[N])}{1+E[N]} S\left(S E[N]+E\left[N^{2}\right]\right) \\
-\frac{2 S E[N]}{1+E[N]}\left(S+1+2\left(-S^{2}+S+1\right) E[N]+(1-S) E\left[N^{2}\right]\right) \\
\gamma_{6}=\left(-2 S^{2}+3 S-1\right) E[N]+S(S-1) P(N \geq 1)+(1-S)^{2} E\left[N^{2}\right] \\
(1+(1-S) E[N])\left(2 E[N] S(1+E[N])+(1+(1-S) E[N]) E\left[(N+1)^{2}\right]\right) \\
+\frac{2 S E[N]}{1+E[N]}\left(\left(S^{2}-2 S+1\right) E[N]+1-S\right) \\
-\frac{2(1+(1-S) E[N])}{1+E[N]}\left(S+1+2\left(-S^{2}+S+1\right) E[N]+(1-S) E\left[N^{2}\right]\right)
\end{gathered}
$$

and

$$
\gamma_{7}=\frac{(1+(1-S) E[N])^{2}}{1+E[N]}-\frac{2(1+(1-S) E[N])}{1+E[N]}\left(\left(S^{2}-2 S+1\right) E[N]+1-S\right) .
$$

When inserting $E[X]=1 /(1-\rho)$ into (15) it follows that determining which of the two service measures that can be estimated most accurately, concerns whether the expression (written as a polynomial in $\rho$ ):

$$
\Lambda(\rho)=\gamma_{1}-\beta_{1}+\left(\gamma_{2}-\beta_{2}\right) \rho+\gamma_{3} \rho^{2}+\left(\gamma_{4}-\beta_{3}\right) \rho^{S-1}+\left(\gamma_{5}-\beta_{4}\right) \rho^{S}+\left(\gamma_{6}-\beta_{5}\right) \rho^{S+1}+\gamma_{7} \rho^{S+2}
$$

is above zero (meaning OFR can be estimated most accurately) or below zero (meaning $V F R$ can be estimated most accurately). As this expression is quite involved, it is difficult to derive specific results. We state two general theorems and a conjecture.

\section{Theorem 1}

$\Lambda(1)=0$. 
Proof: A stringent mathematical proof requires that we show $\sum_{j=1}^{5} \beta_{j}=\sum_{j=1}^{7} \gamma_{j}$. Instead we use a more intuitive argument. When $\rho$ approaches 1 , the order sizes are prohibitively large. Irrespective of the base-stock level $S$, the service measures $O F R$ and $V F R$ are therefore doomed to be zero. This means that both $V\left[D_{O F R}\right]$ and $V\left[D_{V F R}\right]$ will approach 0 as $\rho$ approaches 1 . In particular, it means $\rho=1$ is a double root of the polynomial in the numerator of (18).

Because the constant term in (20) (after possible further reduction) is different depending on whether $S=1$ or $S \geq 2$, it also means that different conclusions can be reached in these two cases.

\section{Theorem 2}

$A$ : When $S=1$ and $P(T \geq L)=1$, then $V F R$ can be estimated most accurately. $B$ : When $S=1$ and $P(T \geq L)<1$, then there exists a threshold value $\hat{\rho}(1)$ that belongs to the open interval $(0,1)$ such that for all values of $\rho<\hat{\rho}(1)$, it is $V F R$ that can be estimated most accurately. $C$ : When $S \geq 2$, then there exists a threshold value $\hat{\rho}\left(2^{+}\right)$that belongs to the open interval $(0,1)$ such that for all values of $\rho<\hat{\rho}\left(2^{+}\right)$, it is $O F R$ that can be estimated most accurately.

Proof: First we consider when $P(T \geq L)=1$. Then $\frac{\Lambda(\rho)}{\rho}=1-2 S \rho^{S-1}+(2 S-1) \rho^{S}$. When $S=$ $1, \Lambda(\rho)$ is negative for all values of $\rho$ in $(0,1)$. Thus, statement $\mathrm{A}$ is verified. When $S \geq 2$, we get $\frac{\Lambda(\rho)}{(1-\rho) \rho}=\sum_{i=0}^{S-2} \rho^{i}-(2 S-1) \rho^{S-1}$ from which we can establish statement $\mathrm{C}$ in the case $P(T \geq$ $L)=1$. Then we consider when $P(T \geq L)<1$. When $S=1$, it holds that $\beta_{1}+\beta_{3}=\gamma_{1}+\gamma_{4}$, while $\beta_{2}+\beta_{4}-\gamma_{2}-\gamma_{5}=\frac{E[N]}{1+E[N]}$. Therefore, the constant term in $\Lambda(\rho) / \rho$ is negative. Thus, statement B is verified. In case $S \geq 2$ the constant term of $\Lambda(\rho)$ is $\gamma_{1}-\beta_{1}=2(E[N]-P(N \geq 1))$, which is positive. This verifies statement $\mathrm{C}$ in the case $P(T \geq L)<1$.

Based on our numerical investigations we state the following conjecture:

\section{Conjecture}

Except when $S=1$ and $P(T \geq L)=1$, the polynomial $\Lambda(\rho)$ has exactly one root in open interval $(0,1)$.

One drawback of Theorem 2 and the Conjecture is that here one looks at all possible combinations of $P(T<L), S$ and $\rho$. It is, however, more interesting to examine the combinations that give rise to reasonable (say, in the range from $90 \%$ to $98 \%$ ) service levels. This is done in the following section. 


\section{Numerical results}

We first consider the case where $P(T<L)=0.2$ (for instance by letting $L=5$ and $T$ be uniformly distributed between 4 and 9 ). We let $\rho$ vary over its relevant range by assigning it the values: $0.1,0.2, \ldots, 0.9$. For each value of $\rho$ we then decide a value of $S$ such that a prespecified level of OFR and VFR is achieved. First this level is $90 \%$ and then it is $95 \%$. In this numerical study we also investigate an alternative service measure, namely the per-cycle variants of $O F R$ and $V F R$ which can be expressed mathematically as:

$$
1-O F R_{\text {Cycle }}=E\left[\frac{O_{0}+\sum_{i=1}^{N} O_{i}}{1+N}\right]
$$

and

$$
1-V F R_{\text {Cycle }}=E\left[\frac{B_{0}+\sum_{i=1}^{N} B_{i}}{X_{0}+\sum_{i=1}^{N} X_{i}}\right] .
$$

At present we for those two service measures, do not know how to mathematically derive measures for how much actual realisations will deviate from the expected values. Therefore we collect this information by using a simulation program, developed in Visual Basic for Excel. The results of the numerical study are summarized in Tables 1 and 2.

\section{$<$ Tables 1 and 2 about here $>$}

Note that the entries in the first two rows are identical. This is due to the fact that we have to choose an integer value of $S$. For all the cases of the two tables we have $\Lambda(\rho)>0$, which means that, following our definition, it is OFR that can be estimated most accurately. The conclusion is different when considering the per-cycle service measures. First, one should note that even under (9) the two per-cycle measures do not give the same result. They are higher than their long-run average counterparts (this is also proven in [2] for the volume fill rate service measure when $P(T>L)=1$ ). Secondly (and most importantly in relation to the theme of this paper), it is $V F R_{\text {Cycle }}$ that can be estimated most accurately as its confidence interval is the narrowest. In order to add to the generality of these findings, we repeat the numerical study for the case where $P(T<L)=0.6$ (for instance by letting $L=10$ and $T$ be uniformly distributed between 7 and 12). The results are summarized in Tables 3 and 4.

$$
<\text { Tables } 3 \text { and } 4 \text { about here }>
$$

Again we see the same pattern of results as when examining Tables 1 and 2.

\section{Conclusions}

The principal goal of the paper is to analyze which service measure that ex post will deviate least from its ex ante prediction. The paper provides a mathematical derivation of the length 
of the confidence intervals that one will get if the (long-run average) service measures order fill rate $(O F R)$ and volume fill rate $(V F R)$ are estimated in a simulation by using the regenerative method. Based on numerical experiments, our conclusion is that of the two service measures it is $O F R$ that can be estimated most accurately, as its confidence interval is the narrowest. A crucial element in the mathematical analysis is the low-frequency assumption (1). So it could be interesting to explore what happens to the result when this assumption is relaxed. As a by-product of the main analysis we have also collected data on the per-cycle variants of $O F R$ and $V F R$, denoted $O F R_{\text {Cycle }}$ and $V F R_{\text {Cycle }}$ respectively. Here it turns out that it is $V F R_{\text {Cycle }}$ that can be estimated most accurately. It could be interesting to examine under which conditions one could make mathematical derivations, in the same spirit as in this paper, on the variation of $O F R_{C y c l e}$ and $V F R_{\text {Cycle }}$ that one would encounter ex post.

\section{References}

1. Chen, F.Y., Krass, D., 2001. Inventory models with minimal service level constraints. European Journal of Operational Research, 134, 120-140.

2. Chen, J., Lin, D.K.J., Thomas, D.J., 2003. On the single item fill rate for a finite horizon. Operations Research Letters, 31, 119-123.

3. Croston, J.D., 1972. Forecasting and stock control for intermittent demand. Operational Research Quarterly, 23, 289-303.

4. Larsen, C., Thorstenson, A., 2007. A comparison between the order and the volume fill rate for a base stock system under a compound renewal demand process. Journal of the Operational Research Society, doi:10.1057/palgrave.jors.2602407.

5. Law, A.M., Kelton, W.D., 1991. Simulation modelling \& analysis (2nd edition). New York: McGraw-Hill.

6. Schultz, C.R., 1987. Forecasting and inventory control for sporadic demand under periodic review. Journal of the Operational Research Society, 38, 453-458.

7. Silver, E.A., Pyke, D.F., Peterson, R., 1998. Inventory management and production planning and scheduling (3rd edition). New York, Wiley.

8. Thomas, D.J., 2005. Measuring item fill-rate performance in a finite horizon. Manufacturing \& Service Operations Management, 7, 74-80.

9. Ward, J.B., 1978. Determining reorder points when demand is lumpy. Management Science, 24, 623-632. 


\section{Tables}

\begin{tabular}{|r|r|r|r|r|r|}
\hline $\boldsymbol{\rho}$ & $\boldsymbol{S}$ & $(\mathbf{1 0})$ & $\boldsymbol{\Lambda}(\boldsymbol{\rho})$ & $\boldsymbol{E s t ~ O F R}_{\text {cycle }}$ & $\boldsymbol{E s t ~ V F R}_{\text {cycle }}$ \\
\hline 0.1 & 2 & 0.0460 & 0.09013 & $0.028822(0.002583)$ & $0.018455(0.001598)$ \\
\hline 0.2 & 3 & 0.0272 & 0.18432 & $0.017995(0.002187)$ & $0.008653(0.001029)$ \\
\hline 0.3 & 3 & 0.0648 & 0.16723 & $0.047090(0.003585)$ & $0.022743(0.001708)$ \\
\hline 0.4 & 4 & 0.0563 & 0.28134 & $0.041732(0.003437)$ & $0.017789(0.001478)$ \\
\hline 0.5 & 5 & 0.0625 & 0.37031 & $0.051196(0.003874)$ & $0.019814(0.001545)$ \\
\hline 0.6 & 6 & 0.0840 & 0.39390 & $0.065545(0.004411)$ & $0.025393(0.001804)$ \\
\hline 0.7 & 8 & 0.0972 & 0.45598 & $0.078280(0.004824)$ & $0.029676(0.001971)$ \\
\hline 0.8 & 13 & 0.0907 & 0.63797 & $0.074840(0.004756)$ & $0.025057(0.001770)$ \\
\hline 0.9 & 27 & 0.0930 & 0.78995 & $0.076345(0.004828)$ & $0.024215(0.001739)$ \\
\hline
\end{tabular}

Table 1: Long-run average service level at least $90 \%$ and $P(T<L)=0.2$

The data in the last two columns are collected by simulation where 10000 cycles are sampled. In parenthesis is stated the half-width of the $95 \%$ confidence interval.

\begin{tabular}{|r|r|r|r|r|r|}
\hline $\boldsymbol{\rho}$ & $\boldsymbol{S}$ & $(\mathbf{1 0})$ & $\boldsymbol{\Lambda}(\boldsymbol{\rho})$ & $\boldsymbol{E s t ~ O F R}_{\text {Cycle }}$ & $\boldsymbol{E s t ~ V F R}_{\text {Cycle }}$ \\
\hline 0.1 & 2 & 0.0460 & 0.09013 & $0.028822(0.002583)$ & $0.018455(0.001598)$ \\
\hline 0.2 & 3 & 0.0272 & 0.18432 & $0.017995(0.002187)$ & $0.008653(0.001029)$ \\
\hline 0.3 & 4 & 0.0232 & 0.30730 & $0.016043(0.002072)$ & $0.006778(0.000878)$ \\
\hline 0.4 & 5 & 0.0256 & 0.43411 & $0.019123(0.002351)$ & $0.007034(0.000880)$ \\
\hline 0.5 & 6 & 0.0344 & 0.53262 & $0.027010(0.002792)$ & $0.009510(0.001016)$ \\
\hline 0.6 & 8 & 0.0347 & 0.71082 & $0.026947(0.002851)$ & $0.008917(0.001001)$ \\
\hline 0.7 & 11 & 0.0384 & 0.87932 & $0.030963(0.003088)$ & $0.009861(0.001070)$ \\
\hline 0.8 & 17 & 0.0417 & 0.99643 & $0.032091(0.003174)$ & $0.009390(0.001035)$ \\
\hline 0.9 & 34 & 0.0488 & 1.22202 & $0.037692(0.003405)$ & $0.010773(0.001097)$ \\
\hline
\end{tabular}

Table 2: Long-run average service level at least $95 \%$ and $P(T<L)=0.2$

The same comments as made under Table 1 apply here. 


\begin{tabular}{|r|r|r|r|r|r|}
\hline $\boldsymbol{\rho}$ & $\boldsymbol{S}$ & $(\mathbf{1 0})$ & $\boldsymbol{\Lambda}(\boldsymbol{\rho})$ & $\boldsymbol{E s s t ~ O F R}_{\text {Cycle }}$ & $\boldsymbol{E s s t ~ V F R}_{\text {Cycle }}$ \\
\hline 0.1 & 3 & 0.0172 & 1.99559 & $0.011249(0.001347)$ & $0.007289(0.000853)$ \\
\hline 0.2 & 3 & 0.0656 & 1.69856 & $0.043794(0.002723)$ & $0.028728(0.001746)$ \\
\hline 0.3 & 4 & 0.0535 & 2.16499 & $0.036415(0.002588)$ & $0.021055(0.001469)$ \\
\hline 0.4 & 5 & 0.0563 & 2.53363 & $0.041384(0.002839)$ & $0.021800(0.001472)$ \\
\hline 0.5 & 6 & 0.0719 & 2.64961 & $0.053907(0.003218)$ & $0.028000(0.001682)$ \\
\hline 0.6 & 8 & 0.0705 & 3.18050 & $0.050474(0.003138)$ & $0.023512(0.001614)$ \\
\hline 0.7 & 11 & 0.0757 & 3.58512 & $0.054043(0.003302)$ & $0.026972(0.001679)$ \\
\hline 0.8 & 16 & 0.0957 & 3.52386 & $0.070003(0.003787)$ & $0.032981(0.001901)$ \\
\hline 0.9 & 33 & 0.0989 & 3.98570 & $0.076344(0.003921)$ & $0.035345(0.001959)$ \\
\hline
\end{tabular}

Table 3: Long-run average service level at least $90 \%$ and $P(T<L)=0.6$

The same comments as made under Table 1 apply here.

\begin{tabular}{|r|r|r|r|r|r|}
\hline $\boldsymbol{\rho}$ & $\boldsymbol{S}$ & $(\mathbf{1 0})$ & $\boldsymbol{\Lambda}(\boldsymbol{\rho})$ & $\boldsymbol{E s t ~ O F R}_{\text {cycle }}$ & $\boldsymbol{E s t ~ V F R}_{\text {Cycle }}$ \\
\hline 0.1 & 3 & 0.0172 & 1.99559 & $0.011249(0.001347)$ & $0.007289(0.000853)$ \\
\hline 0.2 & 4 & 0.0170 & 2.43589 & $0.011173(0.001345)$ & $0.006448(0.000766)$ \\
\hline 0.3 & 5 & 0.0194 & 2.96869 & $0.013572(0.001549)$ & $0.007098(0.000805)$ \\
\hline 0.4 & 6 & 0.0262 & 3.39385 & $0.018868(0.001907)$ & $0.009014(0.000904)$ \\
\hline 0.5 & 7 & 0.0406 & 3.53125 & $0.029090(0.002374)$ & $0.014186(0.001157)$ \\
\hline 0.6 & 9 & 0.0464 & 3.99287 & $0.032713(0.002499)$ & $0.015672(0.001223)$ \\
\hline 0.7 & 13 & 0.0421 & 4.69886 & $0.030531(0.002428)$ & $0.014000(0.001151)$ \\
\hline 0.8 & 20 & 0.0461 & 5.57941 & $0.032852(0.002606)$ & $0.014198(0.001192)$ \\
\hline 0.9 & 41 & 0.0497 & 6.25970 & $0.036201(0.002686)$ & $0.015480(0.001234)$ \\
\hline
\end{tabular}

Table 4: Long-run average service level at least $95 \%$ and $P(T<L)=0.6$

The same comments as made under Table 1 apply here. 


\section{Figures}

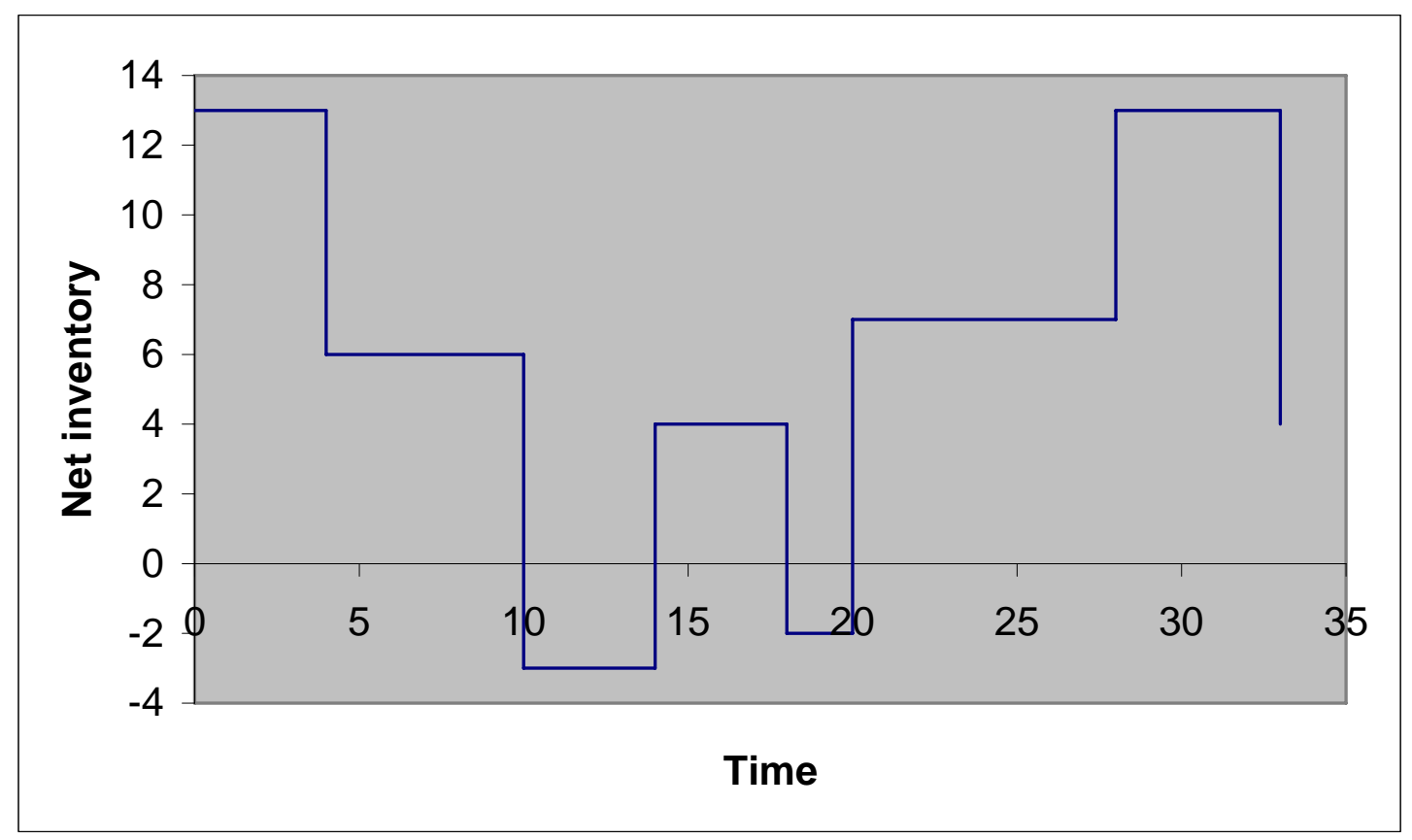

Figure 1: Illustration of a cycle. In the example $S=13$ and $L=10$. The cycle starts in time point 4 and the triggering order is of size $X_{0}=7$ (replenishment received at time point 14). At time point 10 a new order, of size $X_{1}=7$, arrives (replenishment received at time point 20) causing 3 units to be backlogged. At time point 18 a new order of size, $X_{2}=6$, arrives (replenishment received at time point 28) causing 2 units to be backlogged. Because the next order arrives after time point 28 , at time 28 all replenishment orders are received and the net inventory is $S$. The next order (arriving at time point 33) causes the start of a new cycle. 


\section{Appendices}

\section{Appendix A}

In [4] the derivations focus on a point of time where an arbitrary order request arrives. Here $O F R$ and $V F R$ are stated as follows:

$$
1-O F R=P\left(X+D_{L} \geq S+1\right)
$$

and

$$
1-V F R=\frac{1}{E[X]}\left\{P\left(D_{L} \geq S\right) E[X]+\sum_{y=0}^{S-1} P\left(D_{L}=y\right) E[\max \{X-S+y, 0\}]\right\} .
$$

The random variable $D_{L}$ represents the total amount of replenishment orders not yet received at the time point of the order request (or simply denoted lead time demand). When (1) holds, its probability distribution is given as

$$
P\left(D_{L}=y\right)=\left\{\begin{array}{cc}
P(T \geq L) & y=0 \\
P(T<L) P(X=y) & y=1,2, \ldots
\end{array} .\right.
$$

When inserting this probability distribution into the general expressions above, this will lead to $(8)$ and (9).

\section{Appendix B}

$$
\begin{gathered}
E\left[O_{0}\right]=P\left(X_{0} \geq S+1\right)=(1-\rho) \sum_{j=S+1}^{\infty} \rho^{j-1}=\rho^{S} . \\
E\left[O_{1}\right]=P\left(X_{0} \geq S+1\right)+\sum_{j=1}^{S} P\left(X_{0}=j\right) P\left(X_{1} \geq S+1-j\right) \\
=\rho^{S}+(1-\rho) \sum_{j=1}^{S} \rho^{S-1}=S \rho^{S-1}+(1-S) \rho^{S} . \\
=(1-\rho) \frac{d}{d \rho}\left\{\frac{\rho^{S+1}}{1-\rho}\right\}-S \rho^{S}=\frac{(S+1) \rho^{S}-S \rho^{S+1}-S(1-\rho) \rho^{S}}{1-\rho}=\frac{\rho^{S}}{1-\rho} . \\
\left.E\left[B_{0}\right]=\sum_{j=S+1}^{\infty}(j-S) P\left(X_{0}=j\right)=(1-\rho) \rho^{j}\right\}-S \rho^{S} \\
\left.E B_{1}\right]=\sum_{j=S+1}^{\infty} P\left(X_{1}+\max \left\{X_{0}, S\right\}=j\right)(j-S)
\end{gathered}
$$




$$
\begin{gathered}
=P\left(X_{0} \geq S+1\right) E\left[X_{1}\right]+\sum_{j=S+1}^{\infty}(j-S) \sum_{k=1}^{S} P\left(X_{0}=k\right) P\left(X_{1}=j-k\right) \\
=\frac{\rho^{S}}{1-\rho}+\frac{S(1-\rho)}{\rho} \sum_{j=S+1}^{\infty}(j-S)(1-\rho) \rho^{j-1} \\
=\frac{\rho^{S}+S(1-\rho) \rho^{S-1}}{1-\rho}=\frac{S \rho^{S-1}+(1-S) \rho^{S}}{1-\rho} .
\end{gathered}
$$

Inserting these expressions into (7) and (8) verifies that (10) holds.

\section{Appendix C}

We will be slightly more general in order to simultaneously derive $V\left[D_{O F R}\right]$ and $V\left[D_{V F R}\right]$. Let $Y_{0}, Y_{1}, \ldots .$. be a sequence of identically distributed and independent random variables. Now we define other random variables $V_{0}, V_{1}, \ldots .$. where $V_{0}$ is a function of $Y_{0}$, while $V_{i}$ is a function of $Y_{i-1}$ and $Y_{i}$. Thus $V_{1}, V_{2}, \ldots \ldots$ are identically distributed and the all random variables $V_{i}$ and $V_{j}$ with $|i-j| \geq 2$ are independent. $N$ is a non-negative integer-valued random variable which is independent of all the previous random variables. Let

$$
\alpha=\frac{E\left[V_{0}\right]+E\left[\sum_{i=}^{N} V_{i}\right]}{E\left[Y_{0}\right]+E\left[\sum_{i=}^{N} Y_{i}\right]} .
$$

Define the random variable $D$ as

$$
D=V_{0}+\sum_{i=1}^{N} V_{i}-\alpha\left[Y_{0}+\sum_{i=1}^{N} Y_{i}\right]
$$

It holds that

$$
\begin{gathered}
V[D]=E\left[D^{2}\right] \\
=E\left[\left(V_{0}+\sum_{i=1}^{N} V_{i}\right)^{2}\right]+\alpha^{2} E\left[\left(Y_{0}+\sum_{i=1}^{N} Y_{i}\right)^{2}\right]-2 \alpha E\left[\left(V_{0}+\sum_{i=1}^{N} V_{i}\right)\left(Y_{0}+\sum_{i=1}^{N} Y_{i}\right)\right], \\
E\left[\left(V_{0}+\sum_{i=1}^{N} V_{i}\right)^{2}\right] \\
=E\left[\left(V_{0}\right)^{2}\right]+E[N]\left\{2 E\left[V_{0}\right] E\left[V_{1}\right]+E\left[\left(V_{1}\right)^{2}\right]+2 E\left[V_{1} V_{2}\right]-3\left(E\left[V_{1}\right]\right)^{2}\right\}
\end{gathered}
$$




$$
\begin{gathered}
+2 P(N \geq 1)\left\{E\left[V_{0} V_{1}\right]-E\left[V_{0}\right] E\left[V_{1}\right]-E\left[V_{1} V_{2}\right]+\left(E\left[V_{1}\right]\right)^{2}\right\} \\
+E\left[N^{2}\right]\left(E\left[V_{1}\right]\right)^{2} \\
E\left[\left(Y_{0}+\sum_{i=1}^{N} Y_{i}\right)^{2}\right] \\
=E\left[\left(Y_{0}\right)^{2}\right](1+E[N])+\left(E\left[Y_{0}\right]\right)^{2} E[(N+1) N]
\end{gathered}
$$

and

$$
\begin{gathered}
E\left[\left(V_{0}+\sum_{i=1}^{N} V_{i}\right)\left(Y_{0}+\sum_{i=1}^{N} Y_{i}\right)\right] \\
=E\left[V_{0} Y_{0}\right]+E[N]\left\{E\left[V_{0}\right] E\left[Y_{1}\right]-E\left[Y_{0}\right] E\left[V_{1}\right]+E\left[V_{1} Y_{1}\right]+E\left[Y_{1} V_{2}\right]\right\} \\
+E\left[N^{2}\right] E\left[V_{1}\right] E\left[Y_{1}\right] .
\end{gathered}
$$

\section{Appendix D}

Here we specify the elements of $V[D]$ of Appendix $\mathrm{C}$ for the case of the service measure $O F R$. For this case $V_{i}=O_{i}$ and $Y_{i}=1$.

$$
\begin{gathered}
E\left[\left(O_{0}\right)^{2}\right]=E\left[O_{0}\right]=\rho^{S} . \\
E\left[\left(O_{1}\right)^{2}\right]=E\left[O_{1}\right]=S \rho^{S-1}+(1-S) \rho^{S} . \\
E\left[O_{0} O_{1}\right]=E\left[O_{0}\right]=\rho^{S} . \\
E\left[O_{1} O_{2}\right]=\rho^{S-1}+\rho^{S}+\rho^{2 S-1} .
\end{gathered}
$$

\section{Appendix E}

Here we specify the elements of $V[D]$ of Appendix $C$ for the case of the service measure $V F R$. For this case $V_{i}=B_{i}$ and $Y_{i}=X_{i}$. 


$$
\begin{aligned}
& E\left[\left(B_{0}\right)^{2}\right]=\frac{\rho^{S}(1+\rho)}{(1-\rho)^{2}} \\
& E\left[\left(B_{1}\right)^{2}\right]=\frac{S \rho^{S-1}+\rho^{S}+(1-S) \rho^{S+1}}{(1-\rho)^{2}} . \\
& E\left[B_{1} B_{2}\right]=\frac{2 \rho^{S-1}+\rho^{S}+\frac{1}{2} S(S-3) \rho^{2 S-2}-\left(S^{2}-2 S+2\right) \rho^{2 S-1}+\frac{1}{2} S(S-1) \rho^{2 S}}{(1-\rho)^{2}} . \\
& E\left[B_{0} B_{1}\right]=E\left[B_{0}\right] E\left[X_{1}\right]=\frac{\rho^{S}}{(1-\rho)^{2}} \\
& E\left[B_{0} X_{0}\right]=\frac{(S+1) \rho^{S}-(S-1) \rho^{S+1}}{(1-\rho)^{2}} . \\
& E\left[B_{1} X_{1}\right]=\frac{\frac{1}{2} S(S+1) \rho^{S-1}+\left(-S^{2}+S+1\right) \rho^{S}+\left(\frac{S^{2}}{2}-\frac{3}{2} S+1\right) \rho^{S+1}}{(1-\rho)^{2}} . \\
& E\left[B_{2} X_{1}\right]=\frac{\frac{S}{2}(S-1) \rho^{S-1}+\left(-S^{2}+1\right) \rho^{S}+\frac{S}{2}(S-1) \rho^{S+1}}{(1-\rho)^{2}} .
\end{aligned}
$$


L-2008-03 Christian Larsen: Derivation of confidence intervals of service measures in a base-stock inventory control system with low-frequent demand.

L-2008-02 Jens Lysgaard: The Pyramidal Capacitated Vehicle Routing Problem.

L-2008-01 Jens Lysgaard \& Janni Løber: Scheduling participants of Assessment Centres.

L-2007-03 Christian Larsen: Note: Comments on the paper by Rosling (2002).

L-2007-02 Christian Larsen, Claus Hoe Seiding, Christian Teller \& Anders Thorstenson: An inventory control project in a major Danish company using compound renewal demand models.

L-2007-01 Christian Larsen: The $Q(s, S)$ control policy for the joint replenishment problem extended to the case of correlation among item-demands.

L-2006-11 Daniele Pretolani, Lars Relund Nielsen \& Kim Allan Andersen: A note on "Multicriteria adaptive paths in stochastic, time-varying networks".

L-2006-10 Lars Relund Nielsen, Kim Allan Andersen \& Daniele Pretolani: Bicriterion a priori route choice in stochastic time-dependent networks.

L-2006-09 Christian Larsen \& Gudrun P. Kiesmüller: Developing a closed-form cost expression for an $(R, s, n Q)$ policy where the demand process is compound generalized Erlang.

L-2006-08 Eduardo Uchoa, Ricardo Fukasawa, Jens Lysgaard, Artur Pessoa, Marcus Poggi de Aragão, Diogo Andrade: Robust Branch-Cut-and-Price for the Capacitated Minimum Spanning Tree Problem over a Large Extended Formulation.

L-2006-07 Geir Brønmo, Bjørn Nygreen \& Jens Lysgaard: Column generation approaches to ship scheduling with flexible cargo sizes.

L-2006-06 Adam N. Letchford, Jens Lysgaard \& Richard W. Eglese: A Branch-andCut Algorithm for the Capacitated Open Vehicle Routing Problem.

L-2006-05 Ole Mortensen \& Olga W. Lemoine: Business integration between manufacturing and transport-logistics firms.

L-2006-04 Christian H. Christiansen \& Jens Lysgaard: A column generation approach to the capacitated vehicle routing problem with stochastic demands. 
L-2006-03 Christian Larsen: Computation of order and volume fill rates for a base stock inventory control system with heterogeneous demand to investigate which customer class gets the best service.

L-2006-02 Søren Glud Johansen \& Anders Thorstenson: Note: Optimal base-stock policy for the inventory system with periodic review, backorders and sequential lead times.

L-2006-01 Christian Larsen \& Anders Thorstenson: A comparison between the order and the volume fill rates for a base-stock inventory control system under a compound renewal demand process.

L-2005-02 Michael M. Sørensen: Polyhedral computations for the simple graph partitioning problem.

L-2005-01 Ole Mortensen: Transportkoncepter og IT-støtte: et undersøgelsesoplæg og nogle foreløbige resultater.

L-2004-05 Lars Relund Nielsen, Daniele Pretolani \& Kim Allan Andersen: $K$ shortest paths in stochastic time-dependent networks.

L-2004-04 Lars Relund Nielsen, Daniele Pretolani \& Kim Allan Andersen: Finding the $K$ shortest hyperpaths using reoptimization.

L-2004-03 Søren Glud Johansen \& Anders Thorstenson: The $(r, q)$ policy for the lostsales inventory system when more than one order may be outstanding.

L-2004-02 Erland Hejn Nielsen: Streams of events and performance of queuing systems: The basic anatomy of arrival/departure processes, when focus is set on autocorrelation.

L-2004-01 Jens Lysgaard: Reachability cuts for the vehicle routing problem with time windows. 
ISBN 9788778823168

Department of Business Studies

Aarhus School of Business

University of Aarhus

Fuglesangs Allé 4

DK-8210 Aarhus V - Denmark

Tel. +4589486688

Fax +4586150188

www.asb.dk 